Disclosure of interest The authors have not supplied their declaration of competing interest.

http://dx.doi.org/10.1016/j.eurpsy.2017.01.1350
EV1020

\section{Promotion of mental health literacy and mental well-being in a Portuguese unemployed population sample: Effectiveness assessment of a capacity building community-based intersectoral intervention}

M.J. Heitor dos Santos ${ }^{1, *}$, S. Moreira ${ }^{2}$, A. Dinis $^{3}$, A. Virgolino $^{3}$, J. Carreiras ${ }^{3}$, R. Rosa ${ }^{3}$, S. Ambrósio ${ }^{3}$, E. Lopes ${ }^{3}$, T. Fernandes ${ }^{3}$, O. Santos ${ }^{3}$

${ }^{1}$ Faculdade de Medicina da Universidade de Lisboa, Hospital Beatriz Ângelo, Instituto de Medicina Preventiva e Saúde Pública, Instituto de Saúde Ambiental, Departamento de Psiquiatria e Saúde Mental, Lisbon, Portugal

2 Faculdade de Medicina da Universidade de Lisboa, Faculdade de Psicologia da Universidade de Lisboa, Instituto de Medicina Preventiva e Saúde Pública, Instituto de Saúde Ambiental, Lisbon, Portugal

${ }^{3}$ Faculdade de Medicina da Universidade de Lisboa, Instituto de Medicina Preventiva e Saúde Pública, Instituto de Saúde Ambiental, Lisbon, Portugal

* Corresponding author.

Introduction Economic crises have consequences on labor market, with impacts on mental health $(\mathrm{MH})$ and psychological well-being (PWB). We describe the effectiveness of an intervention among unemployed, performed within EEA Grants Healthy Employment project.

Objectives Evaluate the effectiveness of an intervention for $\mathrm{MH}$ literacy, PWB and resilience among unemployed.

Aims $\mathrm{MH}$ and PWB promotion, common mental disorders prevention and inequalities reduction linked to unemployment.

Methods A five modules intervention (life-work balance; impact of unemployment on PWB and MH; stigma; depression and anxiety; health promotion) distributed by 20 hours was developed based on literature reviews and a Delphi panel. It was delivered to unemployees from two public employment centers (PECs). Inclusion criteria: 18-65 years old; registration in PEC for less than a year; minimum of nine years of formal education. Control groups from the same PECs received the care-as-usual. Measures of psychological WB, MH self-reported symptoms, life satisfaction, resilience and mental health literacy were collected through an online survey before and one week after intervention.

Results Overall, 87 unemployed participated, $48 \%$ allocated to the intervention group (IG); 56\% women (21-64 years old), average education was 15 years. Mixed measures ANOVA showed that the interaction between time and group was significant for PWB and MH literacy measures. The IG showed better self-reported PWB and improved $\mathrm{MH}$ literacy after intervention, compared to controls. No significant interactions were found for MH symptoms, life satisfaction and resilience.

Conclusions This study shows the contribution of short-term community-based interventions in increasing MH literacy and PWB among unemployed. 